\section{e0693 COMPARISON OF MYOCARDIAL BRIDGES IMAGING WITH MULTI-SLICE SPIRAL CT AND CORONARY ANGIOGRAPH}

doi:10.1136/hrt.2010.208967.693

Chang Xuewei, Chen Diansen, Ma Huifang, Tian Liping, Zhang Shouyan, Wei Yidong, Wei Jinghan. Department of Cardiology, Luoyang Central Hospital

Objective To assess the diagnostic and clinical value of 64-slice CT coronary angiography (64SCTCA) for evaluation of myocardial bridge $(\mathrm{MB})$ and mural coronary artery (MCA).

Methods A total of 527 patients underwent 64SCTCA. The CT data was reconstructed and post-processed in the work-station. All the cases with $M B$ were submitted to coronary angiography (CAG) studies observing the existence, length and thickness of $\mathrm{MB}$ as well as the stenosis of MCA. The results of CT and CAG were compared and analysed in the end.

Results The 118 of 527 cases with $M B$ segments were found through 64SCTCA. The detection rate is $22.4 \%$. The 45 of 118 cases which were detected by 64SCTCA were found MB positive by CAG. The detection rate is $9.1 \%$. The dates represent significant difference from 64SCTCA and CAG. Statistical significance was established at the $p<0.05$ level. The MB cases were found by 64SCTCA with the mean length of $(6.1 \pm 2.5) \mathrm{mm}$, the mean thickness of $(2.5 \pm 1.6) \mathrm{mm}$ and the mean stenosis rate of $M C$ of $(47.3 \pm 11.3) \%$. The length and the stenosis rate of $\mathrm{MB}$ measured by $\mathrm{CAG}$ represent significant differences from those dates by 64SCTCA $(p<0.05)$.

Conclusion The 64SCTCA can clearly characterise $\mathrm{MB}$ and $\mathrm{MC}$, and has important clinical values.

\section{e0694 EVALUATION OF LEFT HEART FUNCTION IN PATIENTS WITH TYPE 2 DIABETES MELLITUS BY DOPPLER ECHOCARDIOGRAPHY}

doi:10.1136/hrt.2010.208967.694

Hua Wei, Shi Zhongwei, Hu Houda, Zhang Fengru. Rui Jin Hospital of Shanghai Jiao Tong University School of Medicine

Objective To assess left ventricular (LV) structure, LV function, left atrial (LA) structure and LA systolic function by using all the currently available echocardiographic methods in patients with type 2 diabetes mellitus (DM) or impaired glucose tolerance (IGT), with or without coronary artery disease (CAD).

Methods Four groups of patients were evaluated including 35 patients with type 2 DM complicated with chronic CAD, 26 patients with type $2 \mathrm{DM}$ without CAD, 12 patients with IGT without $\mathrm{CAD}$ and 26 healthy controls. All the patients were underwent coronary angiography to diagnose or exclude significant $\mathrm{CAD}$. Echocardiography was performed and a lot of M-mode, 2-DE, pulse wave Doppler and tissue Doppler imaging parameters were measured.

Results All the patients with diabetes or IGT had a normal LV systolic function. However, compared to the control subjects, Am, $\mathrm{Aa}$ and $\mathrm{Em} / \mathrm{Ea}$ were significantly higher $(\mathrm{p}<0.05)$ while Em/Am, Ea and $\mathrm{Ea} / \mathrm{Aa}$ were significantly lower $(\mathrm{p}<0.01)$ in diabetic patients without CAD. Am and Aa were significantly higher $(p<0.01$ and $\mathrm{p}<0.05$, respectively) while $\mathrm{Em} / \mathrm{Am}$ was significantly lower in patients with IGT. Moreover, in patients with type 2 DM or IGT and without CAD, LAVmaxI, LAVminI, LAFF and LAEF were significantly higher. The diabetic patients with CAD had significant higher LVMI, LAVmaxI and LAEF $\left(92.25 \mathrm{~g} / \mathrm{m}^{2} \pm\right.$ $26.96 \mathrm{~g} / \mathrm{m}^{2}$ vs $82.85 \mathrm{~g} / \mathrm{m}^{2} \pm 13.97 \mathrm{~g} / \mathrm{m}^{2}, 31.66 \mathrm{ml} / \mathrm{m}^{2} \pm 7.05 \mathrm{ml} / \mathrm{m}^{2}$ vs $27.45 \mathrm{ml} / \mathrm{m}^{2} \pm 7.19 \mathrm{ml} / \mathrm{m}^{2}, 42.38 \% \pm 9.91 \%$ vs $36.46 \% \pm 5.49 \%$, all $\mathrm{p}<0.05)$ compare to those without CAD.

Conclusions LV diastolic dysfunction, LA dilatation and enhanced LAEF existed already before the occurrence of significant LV systolic dysfunction in patients with type 2 DM or IGT. Patients with diabetes and CAD had more severe LA structural and functional abnormalities. These findings suggests that LA structural and functional abnormalities might be the earliest signs of further cardiac damage in type 2 diabetic patients when significant $\mathrm{CAD}$ is developed.

\section{e0695 THE COMBINED USE OF UROKINASE AND GLYCOPROTEIN IIB/IIIA-TARGETED MICROBUBBLES RECANALIZE RABBIT FEMORAL ARTERY WITH THROMBOTIC OCCLUSIONS}

doi:10.1136/hrt.2010.208967.695

Yuming Mu. Department of the Echocardiograph at the First Affiliated Hospital of Xinjiang Medical University Urumqi

Objective To determine the effect of the combined use of urokinase and glycoprotein IIb/IIIa-targeted microbubbles prepared by direct conjugation method to dissolve the thromb.

Methods Urokinase and RGDS were in conjunction with microbubbles by the direct conjugation method. A total of 42 rabbits with platelet-rich thrombi in the femoral artery were randomised into seven treatment groups ( $n=6)$ : 1) ultrasound alone (US); 2) ultrasound plus non-targeted microbubbles (US $+M$ ); 3) urokinase alone (UK); 4) ultrasound, non-targeted microbubble and urokinase (US $+\mathrm{M}+\mathrm{UK})$; 5) ultrasound plus RGDS microbubble (US+R); 6) RGDS microbubble plus urokinase $(\mathrm{R}+\mathrm{UK})$; and 7) ultrasound, RGDS microbubble and urokinase (US+R+UK). US in diagnostic ultrasound were simultaneously applied over the thrombus up to $30 \mathrm{~min}$. The thrombolytic effect was evaluated at $120 \mathrm{~min}$ post treatment.

Results SonoVue, Urokinase and RGDS were combined successfully. In vitro thrombolysis experiment indicated that the urokinase in the prepared contrast agent had activity $(\mathrm{p}<0.01)$.

Conclusion The combined use of urokinase and glycoprotein IIb/ IIIa-targeted microbubbles is effective in targeting thromb and recanalizing thrombolytic occlusion.

\section{Q0696 INVESTIGATION ON DIFFERENCE OF MECHANICAL PARAMETERS ON CAROTID PLAQUES BETWEEN PATIENTS WITH AND WITHOUT CEREBRALVASCULAR DISEASE USING VELOCITY VECTOR IMAGING}

doi:10.1136/hrt.2010.208967.696

Yin Liu, Mei Zhang, MingJun Xu, Jiang GuiHua, Yun Zhang. Shandong University Qilu Hospital

Objective Our aim was to investigate the difference of mechanical properties of carotid plaques in subjects with and without ischaemic cerebralvascular disease using velocity vector imaging technique.

Methods 162 carotid plaques were detected in 121 subjects. All patients were divided into three groups: patient with acute ischaemic infarction ( $\mathrm{ACI}, \mathrm{n}=48$ ), patients with transient ischaemic attack (TIA, $n=22$ ) and patients without cerebrovascular history (NCD, $\mathrm{n}=51$ ). With velocity vector imaging technique and syngo US Workplace (Siemens), mechanic parameters such as radial velocity (RV), longitudinal strain (LS) and longitudinal strain rate (LSR) were measured on carotid plaques at proximal base (P1), proximal shoulder (P2), top (P3), distal shoulder (P4) and distal base (P5). Morphological parameters including Intima-median thickness, plaque length and lumen diameter was also measured. Receiver operating characteristic curves were constructed from model that associate mechanical and morphological parameters.

Results Higher RV was found in TIA and ACI than that in NCD. In ACI patients, P2-LS was higher than P1-LS, P4-LS and P5-LS 